\title{
Predicting turbulence-induced vibration in axial annular flow by means of large-eddy simulations
}

\author{
Jeroen De Ridder ${ }^{\mathrm{a}}$, Joris Degroote ${ }^{\mathrm{a}}$, Katrien Van Tichelen ${ }^{\mathrm{b}}$, Paul Schuurmans ${ }^{\mathrm{b}}$, Jan Vierendeels ${ }^{\mathrm{a}}$ \\ ${ }^{a}$ Department of Flow, Heat and Combustion Mechanics, Faculty of Engineering and Architecture, Ghent University, Ghent, Belgium \\ ${ }^{b}$ Belgian Nuclear Research Centre, Mol, Belgium
}

\begin{abstract}
Turbulence-induced vibration is typically considered as a type of vibration with one-way coupling between the fluid flow and the structural motion: the turbulence creates an incident force field on the structure, but the structural displacement does not influence the turbulence. It is however challenging to measure the turbulence forcing function experimentally. In this article, the forcing function in annular flow is computed by means of Large-Eddy Simulations. The pressure spectrum is applied to the inner cylinder and the resulting vibration is computed. It is shown that the commonly used multiplication hypothesis does not hold for the present results. The computed spectrum showed an upper limit to the coherence length. The results of these computations are compared to experimental results available in literature and to semi-empirical models. The predicted displacements compared well with experimental results.
\end{abstract}

Keywords: Large-Eddy Simulations, Turbulence-induced Vibration, Annular flow, Axial flow

\section{Introduction}

Turbulence induces vibrations of elastic structures, but the amplitudes of turbulence-induced vibrations are typically small compared to other types of flow-induced vibrations, such as movement- and fluid-instability-induced vibrations (Païdoussis, 2004). As a result, turbulence-induced vibrations normally do not lead to immediate structural failure. However, they can lead to long-term damage such as fatigue and fretting wear.

Turbulence-induced vibration is often treated as a vibration caused by random forces. The random fluid forces are considered to be inherent to a turbulent flow and independent of structural movement. They can either originate from the main flow or from a turbulent boundary layer, which is studied in this paper. Many studies differ from one another in the description of these turbulence forces, which is the main difficulty in the prediction of turbulence-induced vibration.

Many researchers try to find (semi-) empirical characterizations of the pressure field by defining scaling laws for its power spectral density (Chen, 1987; Bull, 1996; Ciappi et al., 2012) and models for its cross-spectral density (Corcos, 1964; Chase, 1987). A comparison between these models has been performed by Graham (1997) and Hambric et al. (2004). In these models, the pressure field is assumed to be axially homogeneous. Ohlmer et al. (1972) showed however that this is not necessarily true and he took streamwise variations into account. However, even with this modification quantitatively correct predictions are not guaranteed. The main reason is that not all model parameters are universally valid. Therefore, these models require experimental data under the correct conditions. Experimental measurements are however often compromised by far-field effects. Furthermore, if measurements of the pressure spectra are necessary, it might often be easier to measure the vibration amplitude instead. Low-wavenumber spectra are for example determined by an inverse procedure in which the excitation field is computed from the actual vibration of a long structure (Evans et al., 2013; Bonness et al., 2010).

Recently, wall-pressure spectra were constructed from numerically computed Reynolds-Averaged Navier-Stokes (RANS) solutions (Lee et al., 2005; Peltier and Hambric, 2007). In these models, the pressure correlation is written as a function of mean shear-turbulence interaction and turbulence-turbulence interaction. The mean shear is readily available in RANS calculations, the turbulence term must however be modeled. A full validation of this wavevectorfrequency spectrum has not yet been performed (Peltier and Hambric, 2007). 


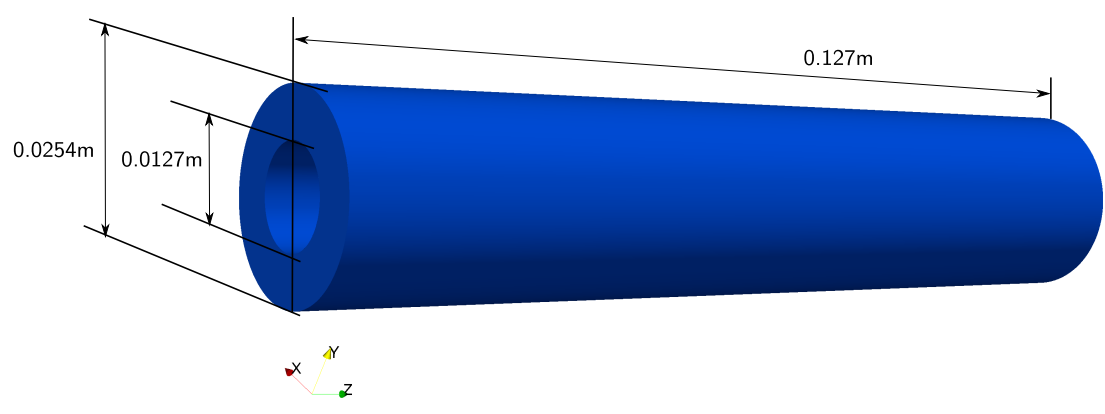

Figure 1: Geometry of the annular fluid domain.

Considering the above mentioned limitations of RANS, it can be assumed that the most accurate method to obtain the pressure field is to directly compute it from a Large-Eddy Simulation (LES) or from a Direct Numerical Simulation (DNS) . Different unsteady simulations such as Unsteady Reynolds Averaged Navier-Stokes (URANS) and DetachedEddy Simulations (DES) are unable to predict the fluctuating pressure field due to the boundary layer as the turbulent fluctuations close to the wall are not resolved, but modeled.

If the pressure field is to be determined in the wake of a bluff body, LES calculations are readily used. These simulations are either at low Reynolds number or use wall-modeled LES simulations for higher Reynolds numbers (Benhamadouche et al., 2009). However, the latter approach is only valid if the turbulent boundary layer is not contributing too much to the turbulence pressure field. Direct Numerical Simulations and Large-Eddy Simulations are starting to be applied to predict the wall pressure spectrum in channel flow (flow in between two flat plates) (Sillero et al., 2014; Gloerfelt and Berland, 2013; Gerolymos et al., 2013).

By contrast, we aim at predicting the turbulence-induced vibrations of a flexible structure inside a narrow rigid pipe. In this annular configuration most numerical research is limited to the computation and analysis of the velocity field (Boersma and Breugem, 2011; Chung et al., 2002). However, Moreno (2000) computed the wall pressure spectrum in an annular flow domain using LES, but the results still suffered from a limited spatial resolution.

To study the turbulence-induced vibrations of a flexible structure inside a narrow rigid pipe, we compute and analyze the incident turbulence forces and their cross-spectral density with a wall-resolved LES. Displacement spectra can then be computed in combination with a modal system, which was previously determined numerically (De Ridder et al., 2013). The power spectral density obtained in this way can be compared to experimental results of Chen and Wambsganss (1972). The computed spectra will also be compared to model spectra predicted by Corcos (1964) and Chase (1987, 1993), which are provided in Appendix.

\section{Methodology}

The setup studied in this article corresponds to the experimental setup described in Chen and Wambsganss (1972). It consists of a brass cylinder with a $0.0127 \mathrm{~m}$ diameter mounted in a small pipe with an inner diameter of $0.0254 \mathrm{~m}$ in which water flows at velocities ranging from 10 to $30 \mathrm{~m} / \mathrm{s}$. In a previous work, modal characteristics of this cylinder were determined numerically and a good comparison was obtained with experimental values (De Ridder et al., 2013). The computational domain is depicted in Figure 1.

In the current paper, the aim is to compute the excitation field at a Reynolds number of 127000 by means of LES instead of the correlations reviewed above. This Reynolds number is calculated with the bulk velocity and the hydraulic diameter. A fixed domain is assumed, corresponding to the one-way coupling assumption. The LES are conducted with OpenFOAM. The filtered incompressible Navier-Stokes equations with constant properties are (with the Einstein summation convention)

$$
\begin{aligned}
& \frac{\partial \overline{u_{i}}}{\partial x_{i}}=0 \\
& \frac{\partial \overline{u_{i}}}{\partial t}+\overline{u_{j}} \frac{\partial \overline{u_{i}}}{\partial x_{j}}=-\frac{1}{\rho} \frac{\partial \bar{p}}{\partial x_{i}}-\frac{\partial \tau_{i j}}{\partial x_{j}}+v \frac{\partial^{2} \overline{u_{i}}}{\partial x_{j} \partial x_{j}} .
\end{aligned}
$$


Table 1: Details of the different meshes used in the grid sensitivity study. The dimensionless spacings are represented by: $\Delta \mathrm{r}^{+}$the radial, $\left(\mathrm{r}_{\mathrm{i}} \Delta \theta\right)^{+}$the spanwise and $\Delta \mathrm{z}^{+}$the streamwise spacing. The integration time refers to the physical simulation time. The ECT refers to the equivalent computing time for $0.1 \mathrm{~s}$ of integration time on a single core.

\begin{tabular}{lcccccccccc}
\hline Name & $\mathrm{N}_{\mathrm{r}}$ & $\mathrm{N}_{\theta}$ & $\mathrm{N}_{\mathrm{z}}$ & $\Delta \mathrm{r}^{+}$ & $\left(\mathrm{r}_{\mathrm{i}} \Delta \theta\right)^{+}$ & $\Delta \mathrm{z}^{+}$ & $\mathrm{L} / \mathrm{D}_{\mathrm{h}}$ & Integration time & $\Delta \mathrm{t}(s)$ & ECT \\
\hline Case A & 80 & 200 & 500 & 3.2 & 93 & 240 & 20 & $0.5 \mathrm{~s}$ & $5 \cdot 10^{-6}$ & 63 days \\
Case B & 140 & 300 & 1000 & 1.1 & 64 & 120 & 20 & $0.3 \mathrm{~s}$ & $2.5 \cdot 10^{-6}$ & 600 days $(2$ years $)$ \\
Case C & 200 & 480 & 800 & 2.1 & 39 & 74 & 10 & $0.7 \mathrm{~s}$ & $2 \cdot 10^{-6}$ & 2200 days $(6$ years $)$ \\
Case D & 200 & 800 & 1100 & 2.1 & 23 & 54 & 10 & $0.25 \mathrm{~s}$ & $1 \cdot 10^{-6}$ & 10000 days $(28$ years $)$ \\
\hline
\end{tabular}

The subgrid-scale stress $\tau_{i j}$ is modeled using a Lagrangian dynamic Smagorinsky model

$$
\tau_{i j}=-2 c_{s}^{2} \Delta^{2}|\bar{S}| \bar{S}_{i j}
$$

with $\bar{S}$ the resolved strain rate tensor, its magnitude $|\bar{S}|=\left(2 \overline{S_{i j} S_{i j}}\right)^{1 / 2}$ and the filter width $\Delta$ equal to the cube root of the cell volume. In the framework proposed by Meneveau et al. (1996), the Smagorinsky coefficient is determined dynamically as the ratio

$$
c_{s}^{2}=\frac{\phi_{L M}}{\phi_{M M}} .
$$

The quantities $\phi_{L M}, \phi_{M M}$ are calculated from two additional transport equations (Meneveau et al., 1996).

At both walls, no-slip boundary conditions are used, which means $u_{i}=0$ and for the pressure $\vec{n} \cdot \nabla p=0$. The inlet and outlet of the domain are periodic. To compensate the pressure drop a constant pressure gradient is added to the momentum equation.

The time discretization is a mixed explicit-implicit formulation of second-order accuracy. The time derivatives are discretized with second order backward differencing. However, the convection term is linearized by using lagged values for the mass flux. As a consequence of the mixed explicit-implicit time scheme, the Courant number is kept below one in all simulations. The spatial discretization is performed with second order central differences. To avoid the odd-even decoupling of pressure, a Rie-Chow like correction is applied.

As can be seen from Equation 2, only the pressure gradient is present in the incompressible Navier-Stokes equations. Consequently, the absolute value is normally determined by the boundary conditions. As the walls are in this case treated by homogeneous Neumann boundary condition and the inlet and outlet are periodic, the pressure is only defined up to a constant value at every time step. Therefore, the pressure is fixed at one point.

\section{Grid sensitivity study}

By definition, an implicitly filtered (by the grid size) LES will be sensitive to the grid size (Bose et al., 2010). As the grid is refined progressively, more flow features will be resolved. It only becomes fully converged when, in the limit of a very fine mesh, it approaches a Direct Numerical Simulation (DNS). This means that the influence of the grid on the solution must be checked.

In this section the influence of grid size on mean flow velocity and on the force spectrum is examined. The mean flow velocity is chosen because its gradient is an important factor in the generation of turbulent pressures. The force density power spectral density is selected as the second criterion because it provides information about the grid sensitivity of statistical quantities. Details about the different simulations are provided in Table 1. Figure 2 illustrates the type of mesh generated, with significant stretching close to the wall.

\subsection{Sensitivity of the mean velocity profile}

The mean velocity profile in the channel is depicted in Figure 3 for a mean channel velocity of $10 \mathrm{~m} / \mathrm{s}$. The left figure shows that the mean velocity reaches a maximum close to the half-width of the channel ( 0.477 times the width), with a value of $11.25 \mathrm{~m} / \mathrm{s}$ (or 1.125 times the mean flow velocity). This is reasonably close to the DNS-results of Chung et al. (2002), who simulated the same geometry but at a lower Reynolds number (8900) and obtained a position of 0.45 

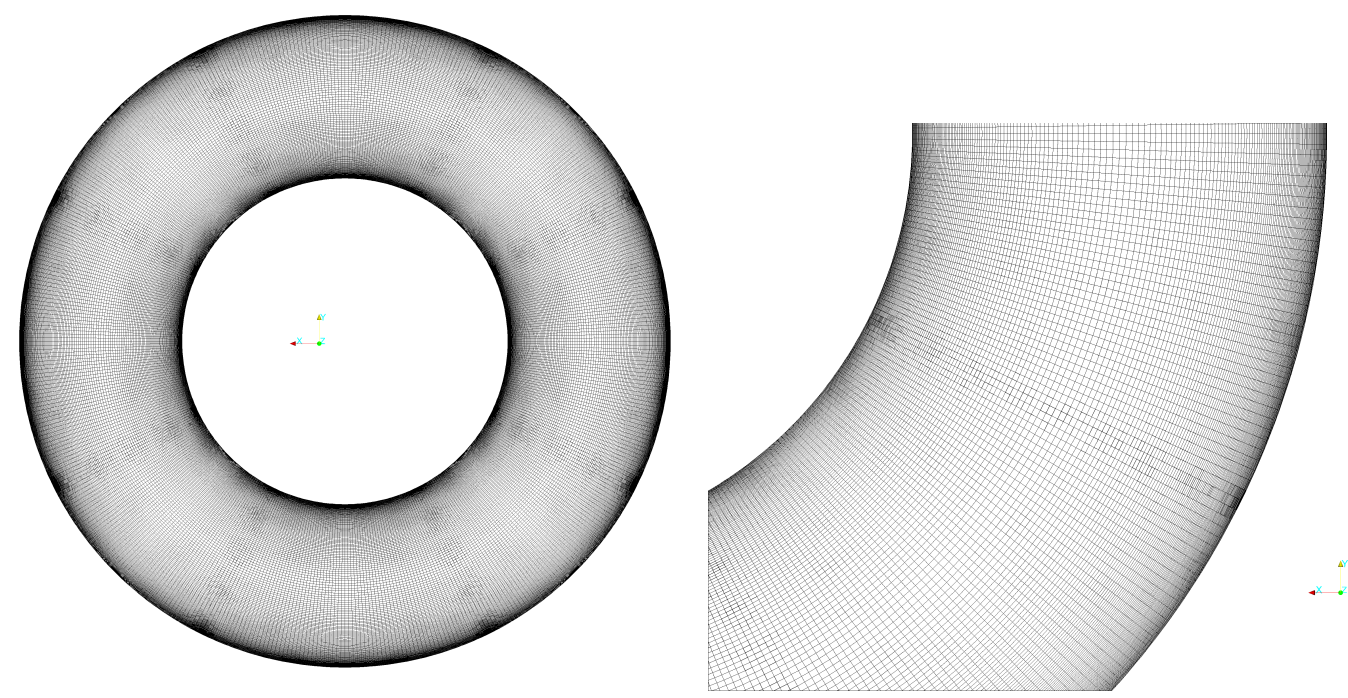

Figure 2: View on the cross-sectional area of the computational mesh.

times the channel width and a maximum of 1.18 times the mean flow velocity. There is also reasonable agreement with experimental values of Nouri et al. (1993), who located the maximal velocity of 1.22 times the bulk velocity at 0.43 times the channel width. The exact location of the maximum velocity in comparison to the point of zero Reynolds shear stresses has been a point of debate in the literature, with some authors stating that they coincide (Boersma and Breugem (2011)), while others state they do not (Chung et al. (2002)). As the current simulations model a (very small) fraction of these shear stresses, this is not analyzed in the present simulations.

Regarding the grid sensitivity, Figure 3 shows that simulation $\mathrm{C}$ is, at least close to the inner wall, reasonably close to the results of the finest simulation (D). Simulations A and B are definitely too coarse. On the right of Figure 3, the dimensionless velocity versus dimensionless wall distance is plotted near the inner wall. The non-dimensional wall shear-stress velocity is calculated from the wall shear stress on the inner wall as $U_{\tau}=\sqrt{\tau_{w} / \rho}$. Distances are made dimensionless by multiplying with $U_{\tau} / v$.

In the viscous sublayer, the mean axial velocity profile can be approximated by the following expression (Boersma and Breugem (2011))

$$
U^{+}=r_{i}^{+} \ln \left(\frac{r}{r_{i}}\right) \approx\left(r^{+}-r_{i}^{+}\right)=y^{+},
$$

with $r_{i}^{+}$the dimensionless distance to the inner wall of the annulus. This is a valid approximation if the distance from the wall is substantially smaller than the radius of the inner cylinder. Note that this approximation means that the effect of the transverse curvature is negligible in the viscous sublayer. The computed mean velocity in Figure 3 shows reasonable agreement with this function.

On the logarithmic sublayer $\left(50<y^{+}<100\right)$, there is less agreement in literature. Generally, the dimensionless velocity is given by

$$
U^{+}=C_{1} \ln \left(f\left(y^{+}\right)\right)+C_{2},
$$

with $f$ a function of $y^{+}$. Willmarth and Yang (1970) take this function equal to $y^{+}\left(1+\frac{y^{+}}{2 r_{i}^{+}}\right)$, while Boersma and Breugem (2011) use $f=\frac{y^{+}}{r_{i}^{+}+y^{+}}$. The former expression has the advantage that it reduces to the well-known logarithmic expression for flow over a flat plate if $r_{i}$ is sufficiently large. Note that in the expression of Boersma and Breugem (2011), the numbers $C_{1}, C_{2}$ are not constants. Instead, they depend on the ratio of the inner wall over external wall radius. As the ratio $r_{i} / r_{o}$ is 0.5 in the present simulations, the velocity profile in this range is expected to be still 

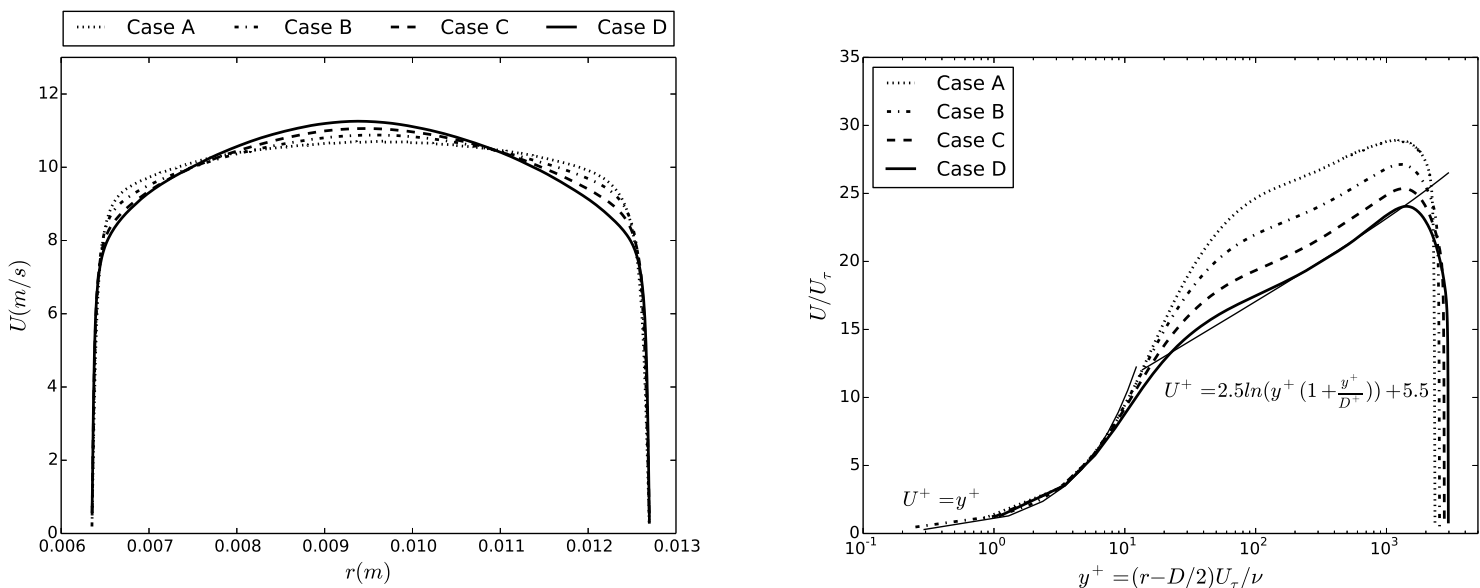

Figure 3: Comparison of the mean velocity between the different computations. The left figure displays the velocity across the channel, while the right figure shows a close-up of the non-dimensional velocity as a function of non-dimensional wall distance.

reasonably close to the profile over a flat plate. Therefore, Equation 6 is plotted in Figure 3, using $\mathrm{C} 1=2.5$ and $\mathrm{C} 2$ $=5.5$, which are the flat-plate constants. These constants are very close to the measured values of $\mathrm{C} 1=2.5$ and $\mathrm{C} 2=$ 5.1 (Willmarth and Yang, 1970).

It can be observed that the computed velocity profile, at least for cases $\mathrm{C}$ and $\mathrm{D}$, is indeed close to this logarithmic law, which was also observed in Moreno (2000). However, there is still a small influence of grid density in the beginning of the log law region $\left(\mathrm{y}^{+} \approx 50\right)$. In this region the slope of the log law is smaller than what is expected from a flat plate. The smaller slope is however consistent with the experimental results in Willmarth et al. (1976). As the $r_{i} / r_{o}$ ratio gets smaller, experimental (Willmarth et al. (1976)) and DNS results (Chung et al. (2002), Boersma and Breugem (2011)) indicate that the slope will decrease further.

\subsection{Sensitivity of the force density autospectrum}

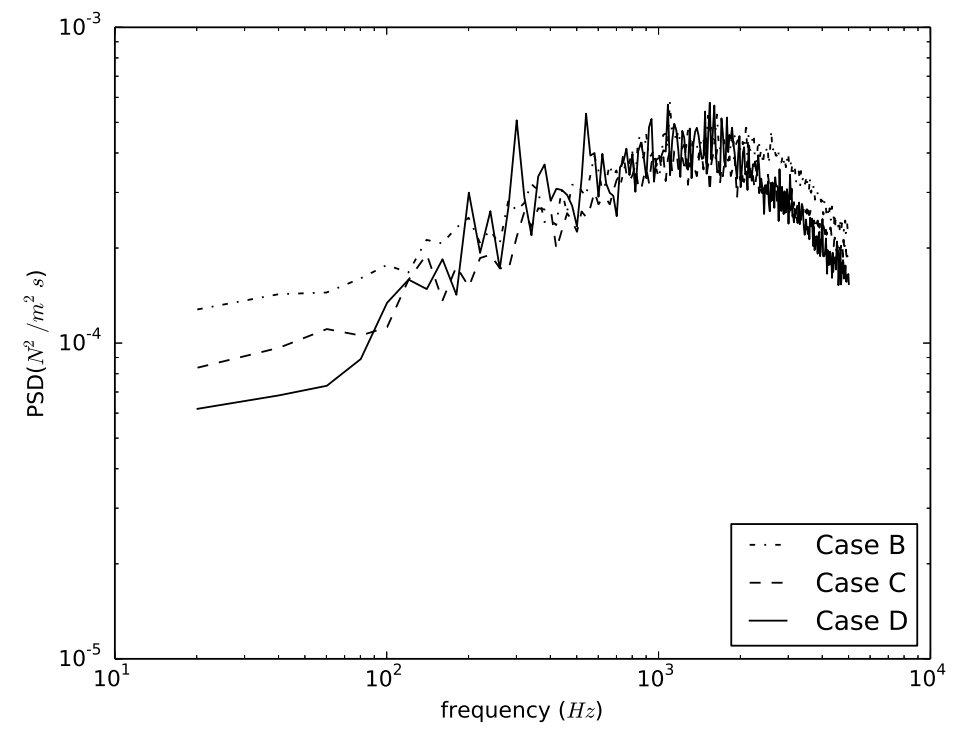

Figure 4: Sensitivity of the power spectral density of force per unit length on the inner cylinder to the grid density. 
Figure 4 displays the sensitivity of the force power spectral density, which will be defined in Section 5 , to the grid density. As the previous section already showed that case A is too coarse, these results are not included in the graph. Simulation B overpredicts the power spectral density in the entire frequency range, compared to simulations C and D. Due to the limited amount of integration time, the latter simulation still shows some scatter. As a result, it is difficult to judge the difference between simulations $\mathrm{C}$ and $\mathrm{D}$. In the low frequency range $(<100 \mathrm{~Hz})$, simulation D predicts a lower power spectral density, but is not clear if this is due to changes in grid resolution or due to a lack of temporal data in simulation D.

In the following paragraphs the results of mesh $\mathrm{C}$ will be used. It is still coarse compared to typical literature values (Choi and Moin, 2012), but the computation on mesh D was too heavy to perform the following analysis of the pressure spectrum. Additionally, this paragraph showed that simulation $\mathrm{C}$ gives reasonable results for the purpose of the present paper

\section{Pressure spectrum}

The forces normal to the inner cylinder are observed to be mainly caused by pressure contributions, which are analyzed in this section. In an incompressible periodic flow simulation, an additional source term is added to the momentum equation in order to balance the shear stress in through-flow direction. This term results in an additional linear pressure variation with axial distance. This pressure gradient due to the shear stresses is not included in the pressure analyzed in this section.

The cross-spectral density of the pressure is computed as follows

$$
\operatorname{CSD}_{p}(\Delta \theta, \Delta z, \omega)=\frac{2}{T\left(N_{\theta}-N_{\Delta \theta}\right)\left(N_{z}-N_{\Delta z}\right)} \sum_{\theta=0}^{N_{\theta}-N_{\Delta \theta}-1} \sum_{z=0}^{N_{z}-N_{\Delta z}-1} P^{*}(\theta, z, \omega) P\left(\theta+N_{\Delta \theta} \Delta \theta, z+N_{\Delta z} \Delta z, \omega\right),
$$

with $N_{x}$ the total number of divisions in direction $x, N_{\Delta x}$ the number of divisions in order to take a step of magnitude $\Delta x, T$ the total sampling time and $P(\theta, z, \omega)$ the Fourier transform of the pressure

$$
P(\theta, z, \omega)=\Delta t \sum_{n=0}^{N_{t}-1} p(\theta, z, n) e^{-\frac{2 \pi k i n}{N_{t}}}, k=0 . . N_{t}-1 \text { and } \omega=\frac{2 \pi k}{T} .
$$

In equation 7, a factor of 2 appears because it is a single-sided spectrum. As the input signal (the pressure) is real-valued, the Fourier spectrum is even. Averages are taken over all spatial directions as the flow is statistically homogenous in the entire simulation domain. The averages in Equation 7 are not crossing the periodic boundary conditions, which results only in small differences.

\subsection{Pressure power spectral density}

The first statistic to analyze is the power spectral density (PSD) which equals the cross-spectral density at zero separation $(P S D(\omega)=C S D(0,0, \omega))$. The power spectral density is plotted in a non-dimensional form $\left(\frac{P S D}{\rho^{2} U^{3} D_{h}}\right)$, used by different authors (Gagnon and Païdoussis, 1994; Curling and Païdoussis, 2003; Chen, 1987), against Strouhal number $\left(S t=\frac{f D_{h}}{U}\right)$ in Figure 5 .

The inner wall spectrum is nearly constant at low frequencies. Around a Strouhal number of 0.5 it starts increasing with increasing frequency until a maximum is reached at a Strouhal number of 2-3 after which it decreases rapidly. The spectrum continues to decrease for higher Strouhal numbers $(>10)$, but as the actual frequencies are not of interest any more to the current research, it is not displayed on the figure. If the high-frequent spectrum was included, it would also be more difficult to see the trends at low frequency, which are of interest in this paper on turbulence-induced vibration.

The agreement with the empirical formula of Chen and Wambsganss (1972) and with Wilson and Jones (1983), which are provided in Appendix A.1, seems to be good, except at low frequencies, which are not in the experimental range. It should however be noted that the original experimental data on which the empirical expression is based, shows a scatter of a factor of 4 for different axial velocities (even when they are plotted in the non-dimensional graph). If one would include data of different, but similar experimental geometries, as plotted in Païdoussis (2004) 


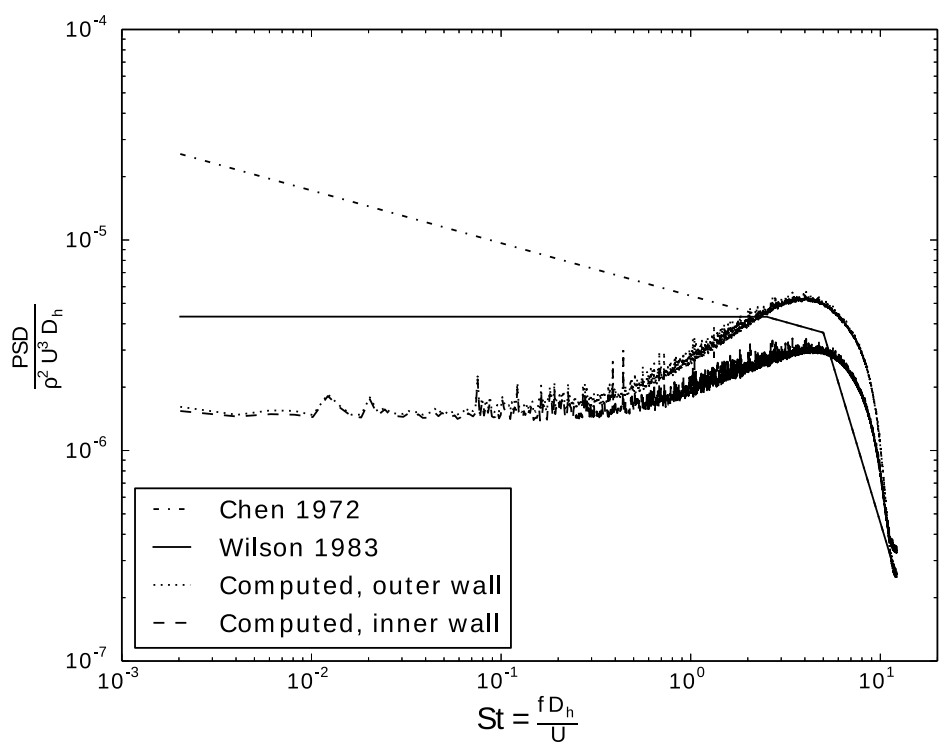

Figure 5: Comparison of non-dimensional pressure power spectral density versus Strouhal number between the simulation results at the inner and outer wall and empirical correlations. The dashed-dotted and solid black lines represent the empirical correlations, the dotted line the result at the outer wall of the annulus and the dashed lines the computed result at the inner wall.

the PSD can be different by a factor of 1000 . This means that even an agreement within one order of magnitude can be considered as good.

The constant low-frequency power spectral density is in agreement with experiments by Wilson and Jones (1983). The peak at $\mathrm{St}=2-3$ is however not seen in that study. In a different study by Curling and Païdoussis (1992), where the pressure spectrum in a cluster of cylinders was measured, a similar peak was visible. This was however only visible on the parts of the cylinder where a significant confinement exists because of the neighboring cylinders. In their measurements the peak was visible at a Strouhal number close to 0.7. The difference in peak Strouhal number might be explained by the difference in geometry (cluster of cylinders versus annular flow).

The spectrum on the outer wall displays a very similar behavior compared to the inner wall spectrum, except that its magnitude at the peak is higher. The peak is also shifted slightly to lower Strouhal numbers. In the experimental results of Wilson and Jones (1983) the low-frequency power spectral density was also higher at the outer wall compared to the power spectral density at the inner wall, however no peaks were visible.

\subsection{Coherence function}

The second statistic characterizing the cross-spectral density is the coherence $\Gamma(\Delta \theta, \Delta z, \omega)$ which is defined as the ratio of the cross-spectral density over the power spectral density:

$$
\Gamma(\Delta \theta, \Delta z, \omega))=\operatorname{CS} D(\Delta \theta, \Delta z, \omega) / P S D(\omega) .
$$

Following the multiplication hypothesis Corcos (1964), the coherence can be written as

$$
\Gamma(\Delta \theta, \Delta z, \omega))=\Gamma(0, \Delta z, \omega) \Gamma(\Delta \theta, 0, \omega) .
$$

Due to this factorization, the further analysis of the coherence is split in its axial and circumferential component. To verify or contradict the validity of the multiplication hypothesis, the analysis of cross-axis components is performed afterwards. 


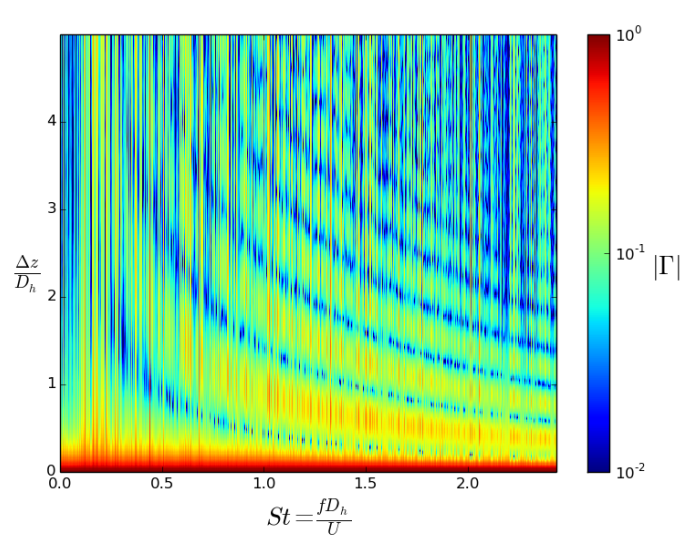

(a) Computed absolute value of coherence

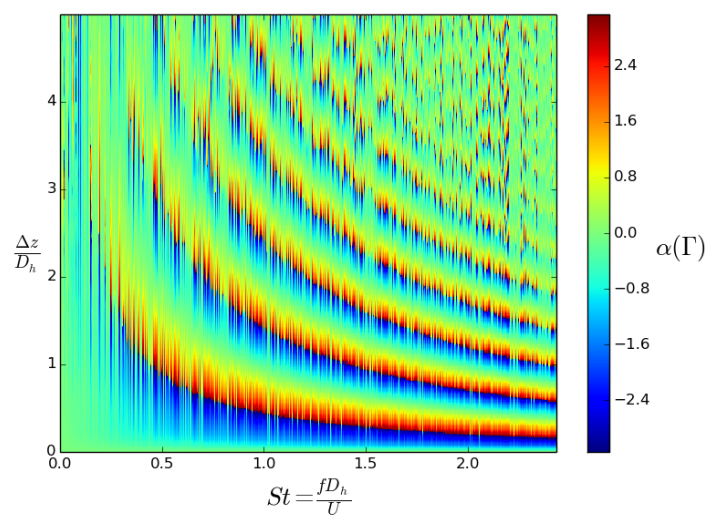

(c) Computed complex angle of coherence

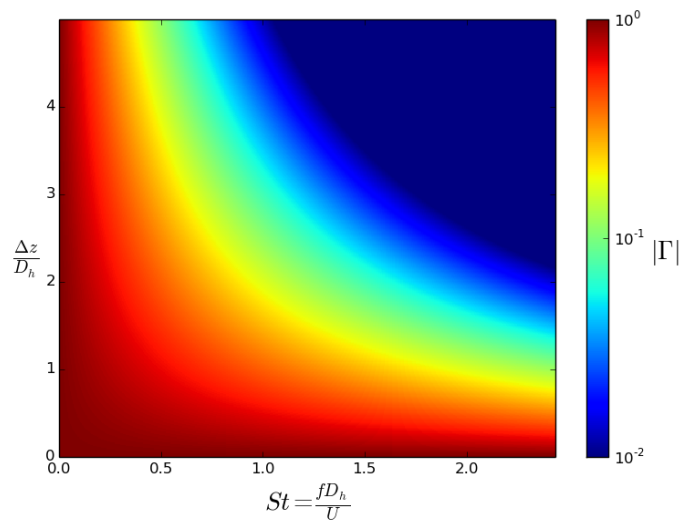

(b) Absolute value of coherence according to Equation 11, $\alpha=0.13$

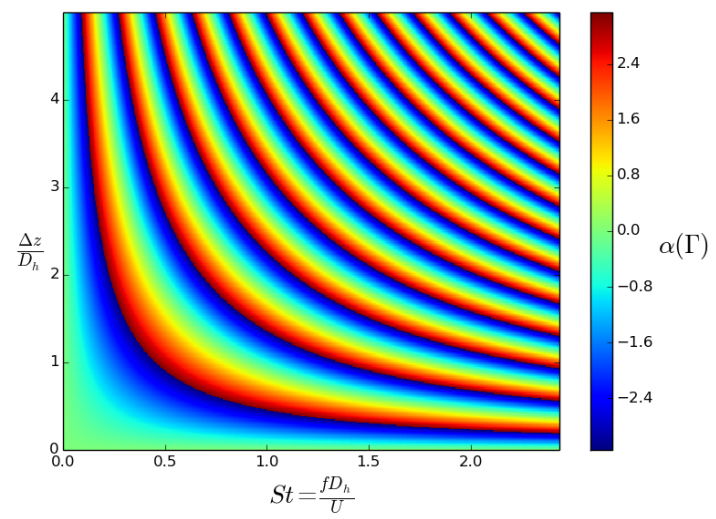

(d) Complex angle of coherence according to Equation 11

Figure 6: Comparison of simulated complex coherence at zero circumferential separation as function of axial separation and frequency and Equation 11. The absolute value of the coherence displays bands with a low value according to the LES-results. The complex angle graph demonstrates that the pressure spectrum is a convected spectrum, in agreement with Equation 11.

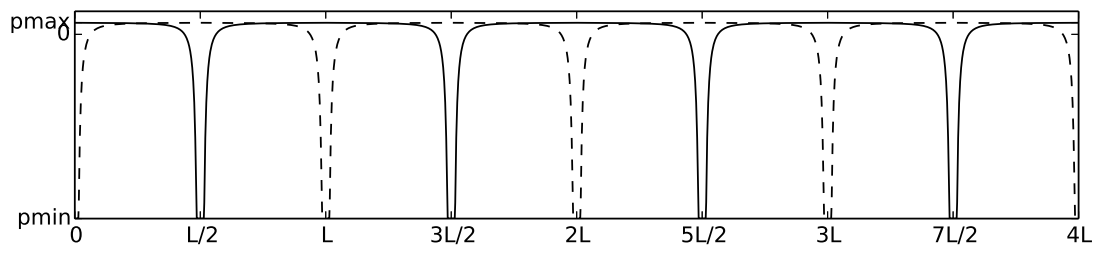

Figure 7: Sketch of the pressure profile (solid line) due to a regular series of irrotational vortices according to a $r^{-2}$ behavior, limited at $p_{\text {min }}$ and the reference level $\left(p_{\max }\right)$ chosen in such a way that the integral of the pressure is zero. The dotted line is the same profile, but shifted over $\pi$ rad. The cross-correlation of both signals will be close to zero. 


\subsubsection{Axial direction}

The coherence function in axial direction is often fitted with complex exponentials

$$
\Gamma(0, \Delta z, \omega))=e^{-\frac{\alpha \Delta z \omega}{U_{c}}} e^{-\frac{i \Delta z \omega}{U_{c}}} .
$$

The first exponential states that the coherence is decaying exponentially such that the coherence equals one at zero separation and zero at infinity. A typical value for the empirical decay factor $\alpha$ is $0.10-0.13$ (confined flow in Curling and Païdoussis (1992)). The second exponential means that pressure fluctuations are transported with the convection velocity $U_{c}$.

The computed absolute value of the coherence as function of axial separation is compared to the empirical spectrum in Figures $6 \mathrm{a}$ and $6 \mathrm{~b}$. There are two major differences between both graphs: firstly, the computed coherence is not decreasing uniformly with increasing distance, instead it displays bands. The minima in between these bands correspond to a phase angle of $\pi$ in Figures $6 \mathrm{c}$ and $6 \mathrm{~d}$. The second difference is that the coherence at low frequencies is lower than what the empirical formula predicts.

Figure $6 \mathrm{~d}$ shows the complex angle which arises due to a convection speed of $U_{c} \approx 0.9 U$. This is in good agreement with the computed complex angle in Figure 6c, indicating that the pressure spectrum is indeed convected with a convection speed $U_{c}$.

The bands in the absolute value of the coherence can be explained by the sketched pressure profiles in Figure 7 . It shows how the pressure profile would look like in a sequence of irrotational vortices occurring at regular intervals. If this profile is transported downstream such that a phase difference of $\pi$ is obtained, one obtains the dashed line profile. It is now straightforward to see that the product of both gives a lower amplitude than at zero phase difference, in contrast with purely sinusoidal signals, where 0 and $\pi$ phase difference gives the same amplitude.

The coherence can also be examined in the time domain, with the instantaneous normalized two-point correlation given by:

$$
\rho_{p p}(\Delta \theta, \Delta z, 0)=\frac{\lim _{\omega_{\max } \rightarrow \infty} \int_{0}^{\omega_{\max }} \mathfrak{R}(\operatorname{CS} D(\Delta \theta, \Delta z, \omega)) \mathrm{d} \omega}{\lim _{\omega_{\max } \rightarrow \infty} \int_{0}^{\omega_{\max }} \operatorname{PS} D(\omega) \mathrm{d} \omega} .
$$

It is plotted as a function of axial distance and at equal circumferential position in Figure 8. In an attempt to separate the low and high frequent contributions to the two-point correlation, it is computed by taking the integral in Equation 12 for $0<\mathrm{St}<0.6,0.6<\mathrm{St}<3.3$ and $3.3<\mathrm{St}$. These intervals correspond to the regions in the pressure power spectral densities of Figure 5 where it is almost constant, increasing to a maximum and decreasing from the maximum. In

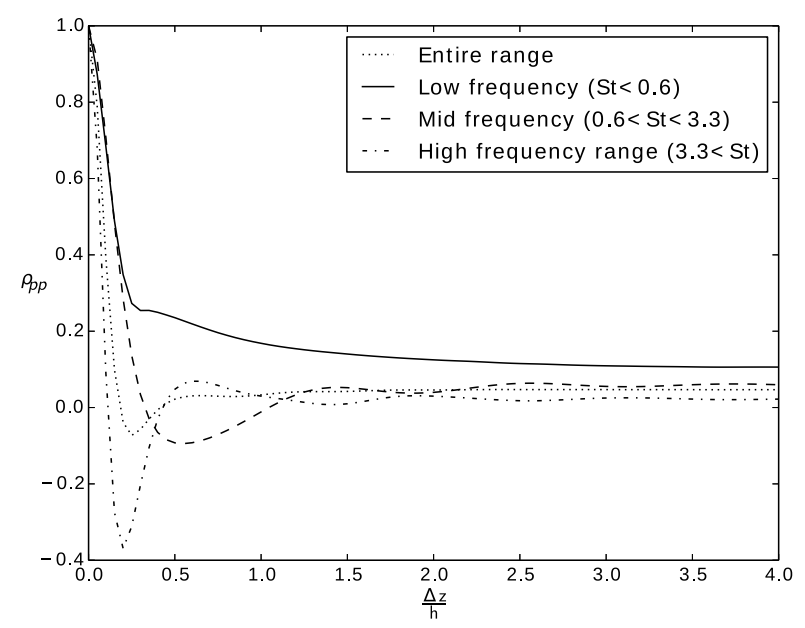

Figure 8: Two-point correlation showing the contribution of different frequency regions to the correlation. 


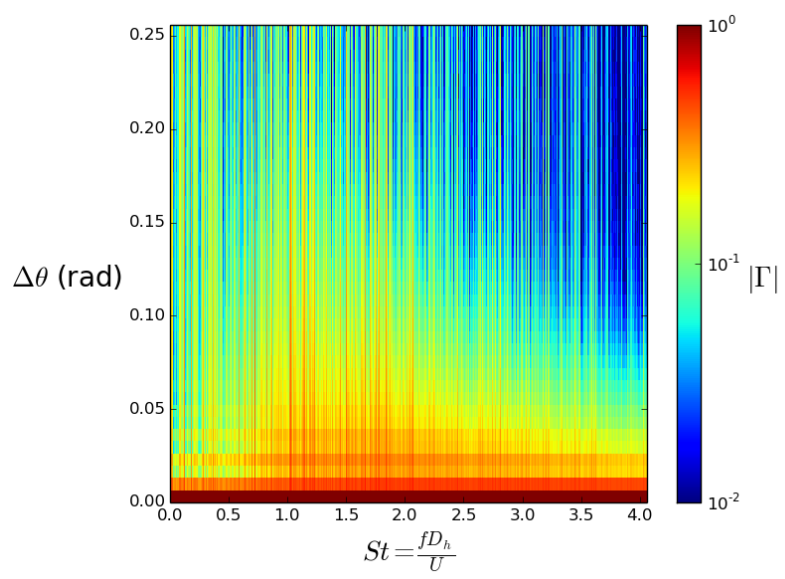

(a) Computed absolute value of circumferential coherence

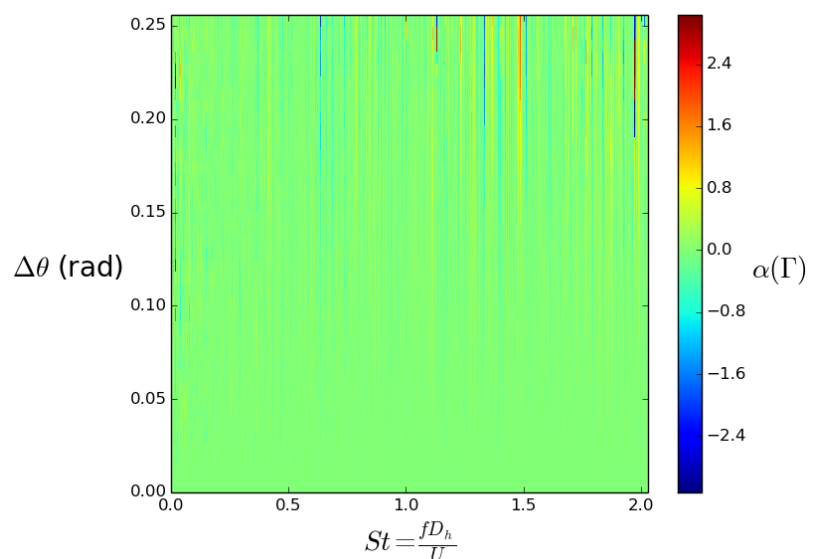

(b) Computed complex angle of coherence

Figure 9: Computed coherence in circumferential direction, showing that fluctuations in this direction are in phase and decaying fast.

reasonable agreement with experimental results of flat-wall boundary layer flow by Bull (1967), the longitudinal correlation decreases strongly until a length of approximately $\Delta z / h=4$, with $h$ being the half-height of the channel $\left(=D_{h} / 4\right)$. The current results also agree reasonably with the measurements of Willmarth and Yang (1970) who measured a correlation length (the integral of the two-point correlation) of 1.31 times the boundary layer height on a body of revolution.

Theoretically, the correlation should drop to zero at large distances. However, in the present results a small amount of far-field correlation remains present at those distances. This can be explained by the solver used. As the flow is incompressible and periodic boundary conditions are used, the pressure is only defined up to a constant in the domain. To fix the absolute pressure level, a reference point in the domain is given zero-pressure. If a fluctuation passes this point, the pressure level in the total domain will change instead of the pressure in this single point. This should however not affect the statistics too much, but it gives a small amount of artificial pressure correlation.

\subsubsection{Circumferential direction}

In circumferential direction, the coherence function is typically assumed to be in phase and can be described (in the high-frequency domain) by:

$$
\Gamma(\Delta \theta, 0, \omega))=e^{-\frac{\beta \Delta \theta R \omega}{U_{c}}},
$$

with $\beta$ an empirical factor equal to 0.644 on flat wall boundary layer flow, as determined by Bull (1967).

Figure $9 \mathrm{~b}$ shows that the phase angle of the circumferential coherence indeed equals zero. The absolute value of the coherence in Figure 9a shows a very fast decrease with increasing distance. The decrease is significantly larger than the one predicted in the empirical formula of Equation 13. At high frequencies this formula might work, but at low frequencies ( $S t$ below one) it predicts a very high correlation for angular distances of $\pi$. As pressure differences arise from vortices in the boundary layer, it is unlikely that a vortex is capable of creating such a correlated pressure, except maybe for a global swirling motion.

The two-point correlation, computed the same way as in the previous section, is plotted in Figure 10. In contrast to the two-point correlation in the axial direction, the difference between the low, middle and high frequency contributions is smaller. The far-field contribution is also an artifact of the way the computations are carried out as explained in the previous section. The short correlation length is in agreement with recent experimental results of Bailey et al. (2008), in which the azimuthal structures and pressures in an annular geometry were measured, although the measurements were restricted to a minimal distance from the wall. 


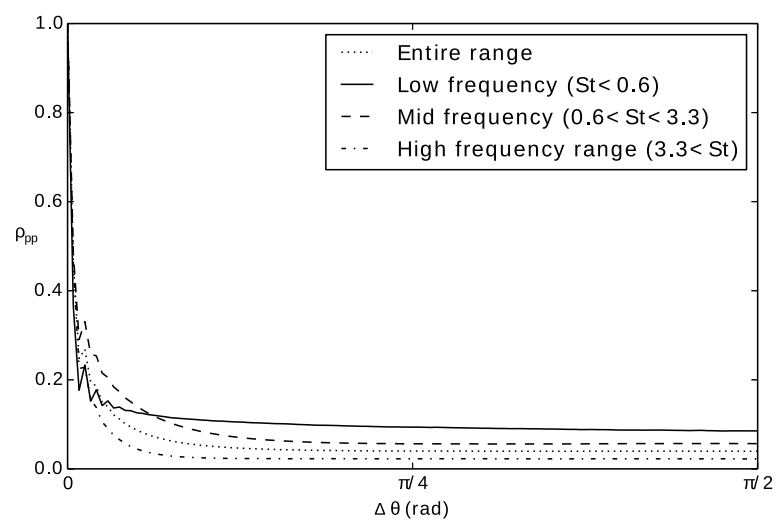

Figure 10: Two-point correlation showing the contribution of different frequency regions to the correlation.

\subsubsection{Mixed direction}

To verify the multiplication hypothesis, the coherence at fixed frequency is plotted as a function of circumferential and axial distance in Figure 11. The absolute value and the complex angle are plotted for St $=0.06,0.6,1.2$ and 1.8. The main purpose of these graphs is to show trends in the coherence function. They should not be used standalone as the absolute value shows some variation with minor frequency shifts, as can also be seen in Figure 6.

The graphs show the evolution of the coherence downstream and along the circumference of the inner cylinder. At the lowest frequency, which is displayed in Figure 11a, the fluctuations still show some coherence, although it decays relatively fast in the circumferential direction. This is probably because the vortices at this frequency become too large to fit inside the geometry.

At higher frequencies (Figures 11c,11e,11g), the coherence decays fast circumferentially at zero axial separation. However, at axial separations, corresponding to a few turn-over times, the spectrum is correlated over a fairly high circumferential range. This is in contradiction with the multiplication hypothesis, which says that the coherence in the circumferential direction should decay similarly at different axial separation.

The bands in Figure 11 stem from the convected nature of the pressure. As the pressure fluctuation (or vortex) is transported, the coherence tends to smear out, both in the axial and the circumferential direction. In this way, the pressure profile has coherent zones and can trigger structural movement.

\section{Force spectrum at high Reynolds number}

As the lowest-frequency structural modes of a beam, which are the easiest to excite, are independent of the circumferential angle, it is the force density per unit length which will excite the cylinder. The spectrum $S_{f}$ of the force density $(\mathrm{N} / \mathrm{m}) F(x, t)=\int_{0}^{2 \pi} p \vec{n} a \mathrm{~d} \theta$, with $a$ the radius of the inner cylinder can be computed directly by integrating the pressure spectrum along the circumference of the cylinder

$$
S_{f}(\Delta z, \omega)=\int_{0}^{2 \pi} \int_{0}^{2 \pi} C S D\left(\left|\theta_{1}-\theta_{2}\right|, \Delta z, \omega\right) a^{2} \cos \left(\theta_{1}\right) \cos \left(\theta_{2}\right) \mathrm{d} \theta_{1} \mathrm{~d} \theta_{2}
$$

Here, the force cross-spectral density is computed in two steps, as the resulting procedure is faster. First, the Fourier transform of the pressure is integrated to yield the Fourier transform of the linear force,

$$
F_{i}(z, \omega)=\sum_{j=0}^{N_{\theta}-1} P\left(\theta_{j}, z, \omega\right) a \cos (j \Delta \theta-i \Delta \theta) \Delta \theta
$$


(a) $|\Gamma|, S t=0.06$

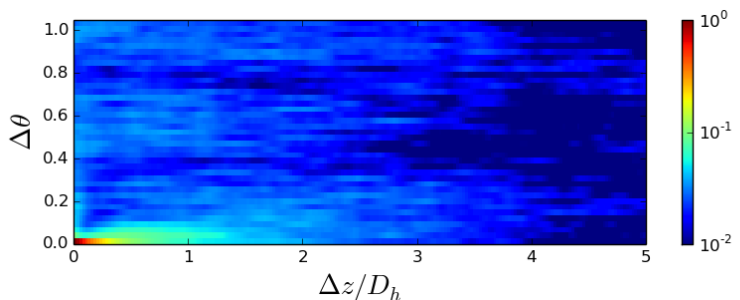

(c) $|\Gamma|, S t=0.6$

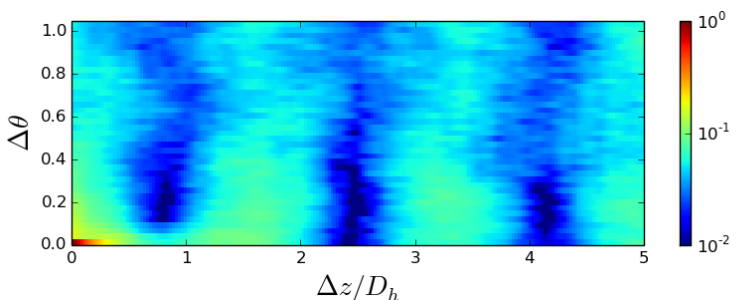

(e) $|\Gamma|, S t=1.2$

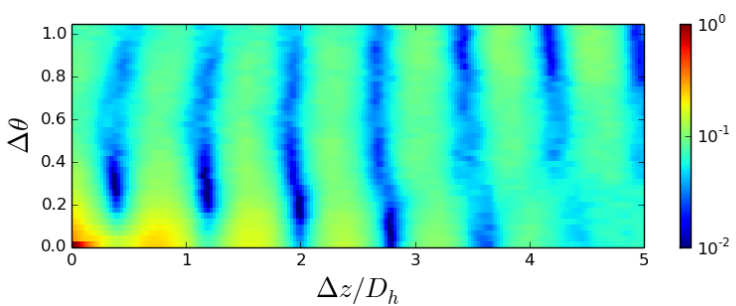

(g) $|\Gamma|, S t=1.8$

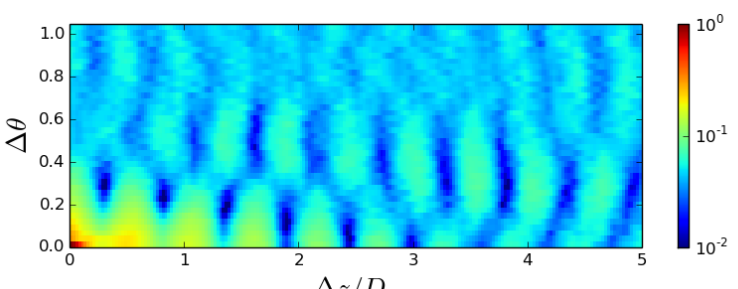

$\Delta z / D_{h}$ (b) $\alpha(\Gamma), S t=0.06$

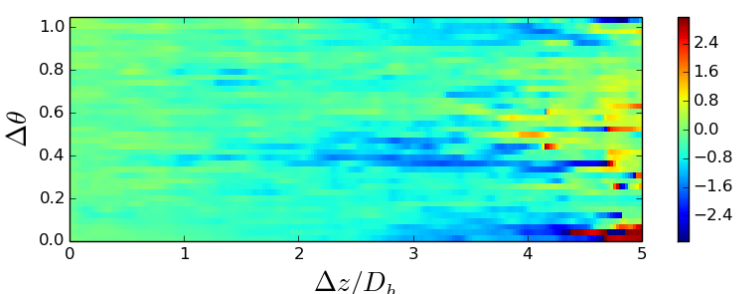

(d) $\alpha(\Gamma), S t=0.6$

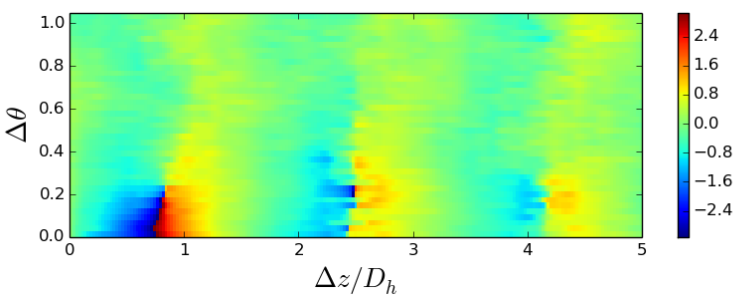

(f) $\alpha(\Gamma), S t=1.2$

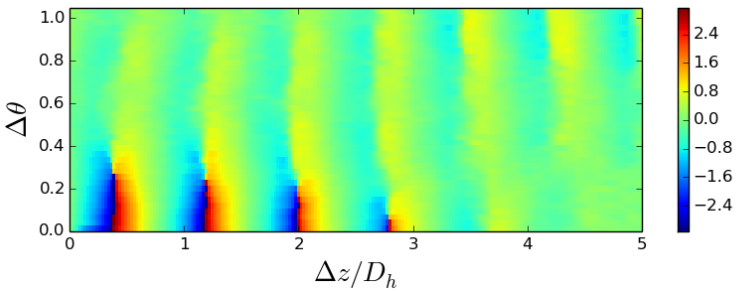

(h) $\alpha(\Gamma), S t=1.8$

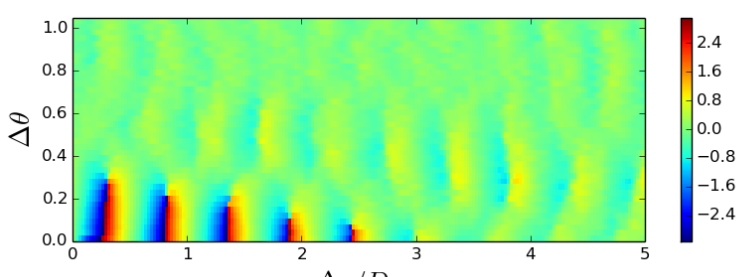

$\Delta z / D_{h}$

Figure 11: Computed coherence at different Strouhal numbers as a function of streamwise and spanwise separation, displaying the convection and decay properties of turbulent wall pressure fluctuations. 
In this equation $F_{i}$ represents the transverse force in direction i. These forces are then correlated with each other

$$
S_{f}(\Delta z, \omega)=\frac{2}{T N_{\theta} N_{z}} \sum_{i=0}^{N_{\theta}-1} \sum_{z=0}^{N_{z}-1} F_{i}^{*}(z, \omega) F_{i}\left(z+N_{\Delta z} \Delta z, \omega\right) .
$$

Note that the reported cross spectral density $S_{f}$ is the average of force cross spectral densities in all directions in the $\mathrm{x}, \mathrm{y}$-plane.

The analysis of the cross-spectral density will be divided in three parts: the power spectral density of the fluctuations, the absolute value of the coherence and the phase of the cross-spectral density. The coherence is defined in the same way as in the previous part, but then based on force density.

\subsection{Power spectral density}

The power spectral density of the force density is shown in Figure 12. At low frequencies the simulated power spectral density shows a slightly decreasing trend with decreasing frequency. At approximately $1000 \mathrm{~Hz}$ a maximum is reached, after which the spectrum decreases with increasing frequency.

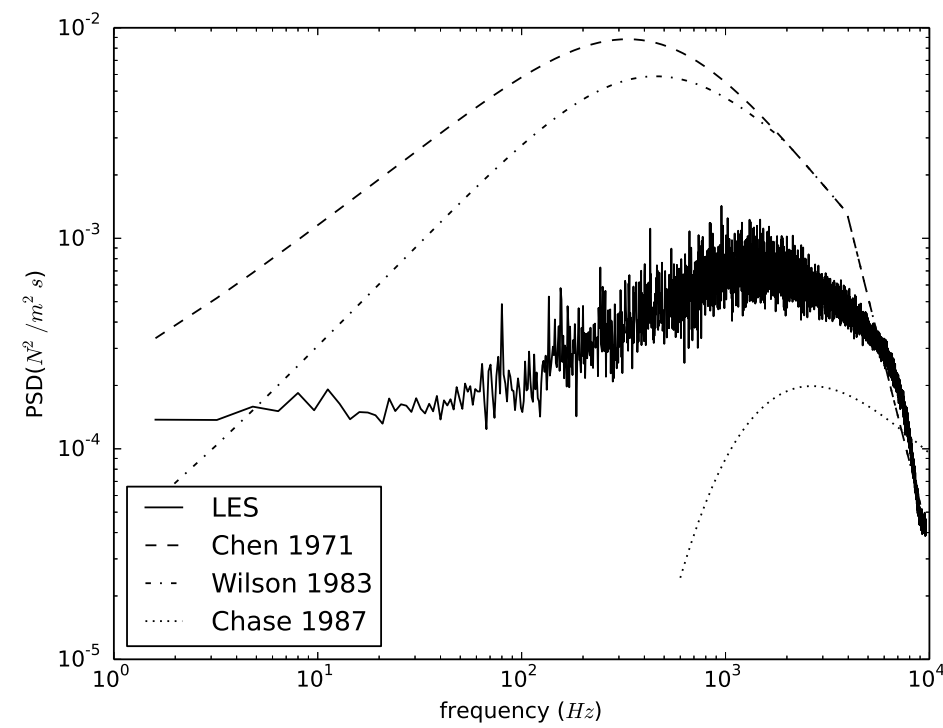

Figure 12: Power spectral density of force per unit length. The solid line represents the results from the current LES-computations, the dashed line represents the empirical results by Chen and Wambsganss (1972), the dashed-dotted line the correction by Wilson and Jones (1983) and the dotted line the predictions by the Chase model.

A reasonable agreement is obtained with the Chase model in the $1-5 \mathrm{kHz}$ range $(S t \approx 1-5)$. In the low frequency range however the Chase model predicts a too quick decrease in power spectral density. The Chase model is commonly used for turbulent pressure spectra on flat plates. However, it predicts too coherent structures in the circumferential direction on a body of revolution, similarly to the Corcos model, as discussed in section 4.2.2. A second reason, why the Chase spectrum is decreasing quickly, is because the wall pressure spectrum follows a quadratic scaling, in agreement with the Kraichnan-Philips theorem. Experimentally however, a wavenumber-white spectrum is found (Evans et al., 2013), in agreement with the current computational results. This part of the spectrum might be caused by the coupling of pressure and wall shear stress spectrum (Chase, 1993).

The experimental pressure power spectral density measurements of Chen (1987) are converted to forces by using the Corcos coherence model. The trends in this spectrum at low frequency correspond much better to the LES-values. The absolute values are however higher than the LES ones. The wavenumber-white low frequency force spectrum is not present in the Corcos / experimental results, although experimentally a wavenumber-white low frequency pressure 
spectrum is found. It is however not visible in the force spectrum because of the circumferential coherence used: as the frequency reduces to low values, the circumferential coherence becomes one and the force, which is an integral of the pressure, becomes zero.

The remaining disagreement between the experimental values and the LES-values can have many causes. Firstly, there is still a considerable scatter on the experimental PSD. Secondly, a Corcos type of coherence is assumed, which might be invalid and finally it could be that a part of the numeric spectrum is dissipated because of the finiteness of the computational domain.

\subsection{The coherence function}

In order to have some structural response the force spectrum needs to be correlated along a certain length. The computed absolute value of the coherence $\Gamma_{f}$, which is a measure for this correlation is plotted in Figure 13a. The right part of this figure shows that isolines of coherence magnitude correspond with hyperbolic lines. In this region, the coherence decays exponentially with increasing frequency and spatial separation. The left part however does not follow this trend. Instead, there is a decrease of the coherence with decreasing frequency.

Already in the phenomenological model of Corcos (1964), the right side of the coherence spectrum is described by an exponential decay as in Equation A.3. The low frequency part of the spectrum, which is important for structural vibration is however not correctly represented by the Corcos model. The high frequent part is in reasonable agreement with the computational results, although the same model gave significantly different results for the pressure coherence.

The decrease of the coherence at the left side of the convective peak, which is located at approximately $1000 \mathrm{~Hz}$, is captured to some extent by the Chase model, which is shown in Figure 13e. The convective peak itself is also very well captured by the Chase model. On the other hand, the Chase model (Equation A.4) predicts a vanishing coherence at low frequencies. The simulations however gave still some amount of coherence, as can be seen at the left side of Figure 13a. As already noted by Chase (1993), the low frequency range might be governed by fluctuations arising from the pressure boundary condition where the boundary pressure is linked to the viscous stress.

A more intuitive understanding to the maximum coherence observed in Figure 13a is that fluctuations are restricted to geometrical sizes and fluctuations can therefore not grow infinitely large, which is the approach followed in Smol'yakov (2006) for example.

To have a structural response, the fluctuations should not only be correlated along a certain length but should also have the right phase angles. The computed phase angles are plotted in Figure 13b. At zero frequency and at zero distance between the points, the phase angle equals zero or $2 \pi$. Away from these boundaries, one can find lines of constant angle at hyperbolic lines.

The hyperbolic isolines of phase angle are recovered in the Chase model (Equation A.4) and in the Corcos model, as plotted in Figures $13 \mathrm{f}$ and 13d. Similarly as the phase angles of the pressure coherence, they can be interpreted as a time delay, which results from convection. The phase angle of the Chase model at low frequencies is out of phase with the computed values, indicating that it is not suitable for these low frequency fluctuations.

\section{Displacements predicted by the current spectra}

The displacement $d$ in any direction normal to the $\mathrm{z}$-axis can be written as a linear combination of modal shapes $d(z, t)=\sum_{\alpha} q_{\alpha}(t) \phi_{\alpha}(z)$, with $\phi_{\alpha}(z)$ the mode shapes. The Fourier transform of the modal displacements $Q_{\alpha}(\omega)$ can be linked to the Fourier transform of the forces $F(z, \omega)$ by

$$
\begin{aligned}
& Q_{\alpha}(\omega)=H_{\alpha}(\omega) \int_{0}^{L} \phi_{\alpha}(z) F(z, \omega) \mathrm{d} z, \\
& H_{\alpha}(\omega)=\frac{1}{m_{\alpha}\left(\omega_{\alpha}^{2}-\omega^{2}+2 \omega \omega_{\alpha} \zeta_{\alpha} i\right)},
\end{aligned}
$$


(a) $\left|\Gamma_{F}\right|$, computed

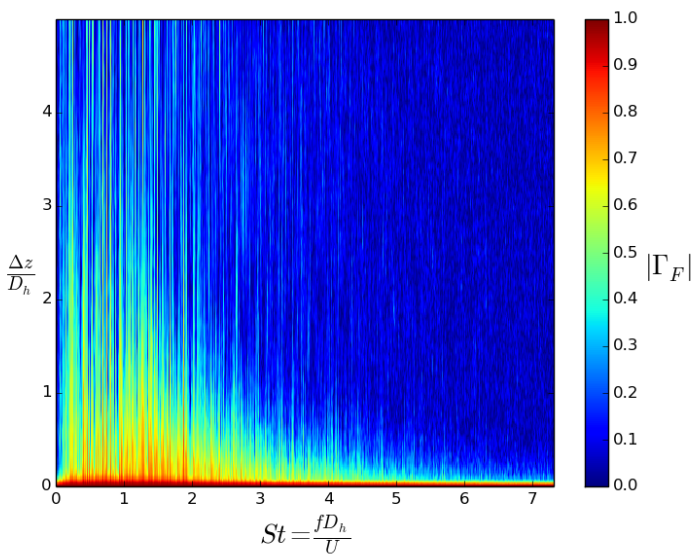

(c) $\left|\Gamma_{F}\right|$, Corcos model

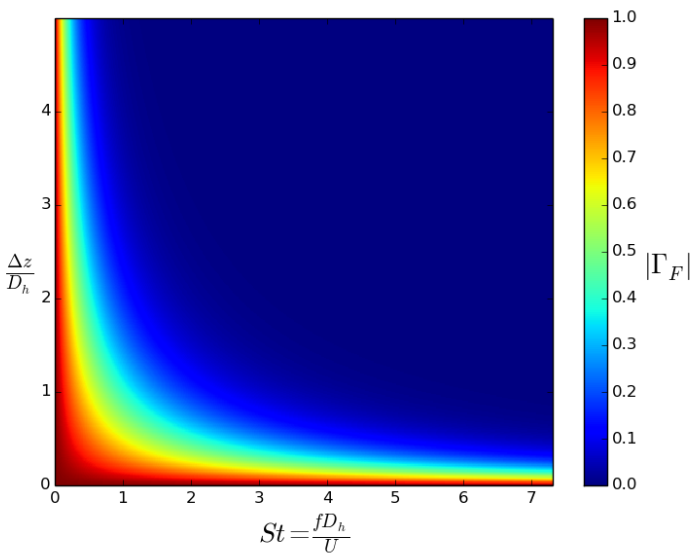

(e) $\left|\Gamma_{F}\right|$, Chase model

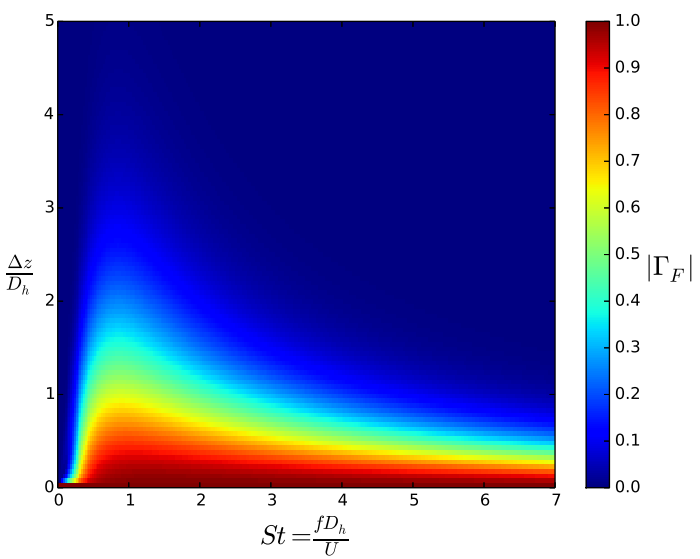

(b) $\alpha\left(\Gamma_{F}\right)$, computed

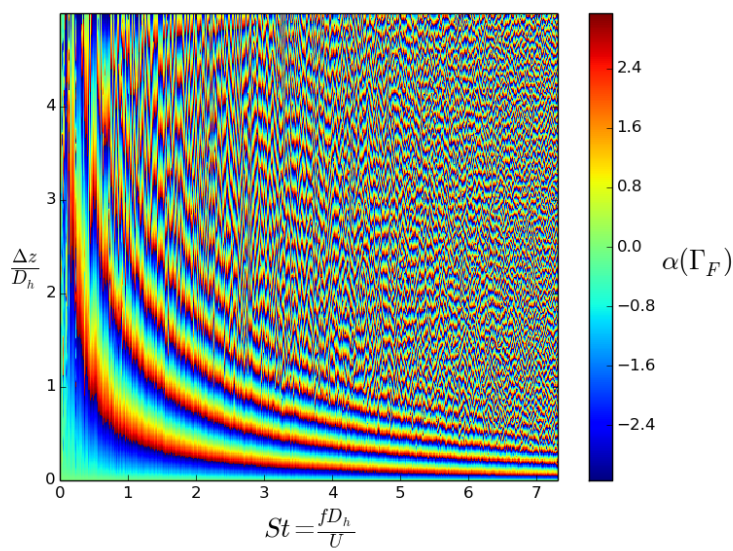

(d) $\alpha\left(\Gamma_{F}\right)$, Corcos model

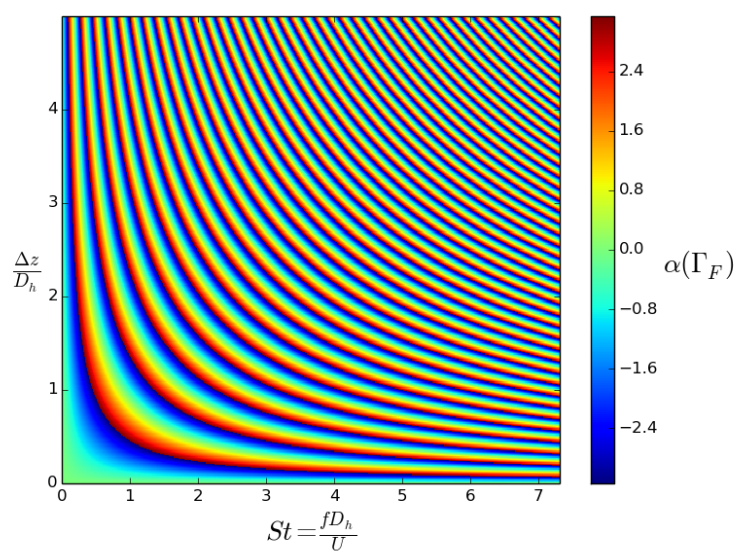

(f) $\alpha\left(\Gamma_{F}\right)$, Chase model

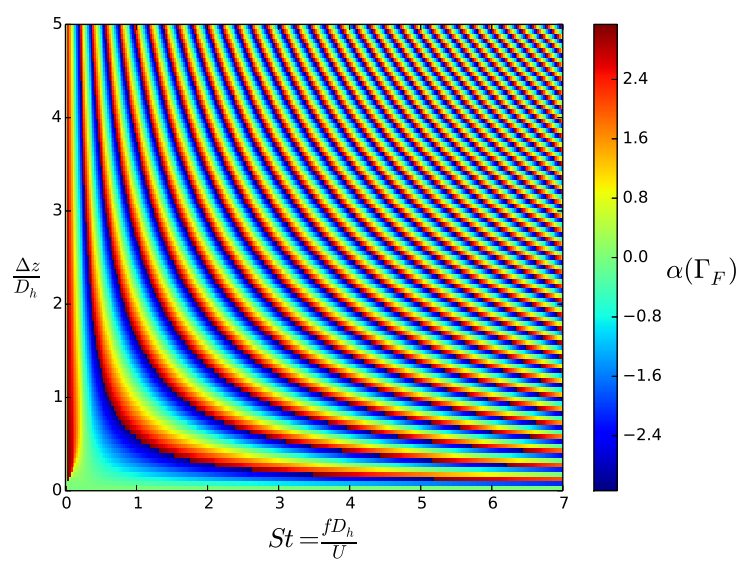

Figure 13: Comparison of the force coherence resulting from the LES-calculations, according to Chen (1987), who used the Corcos model, and Chase (1987) 


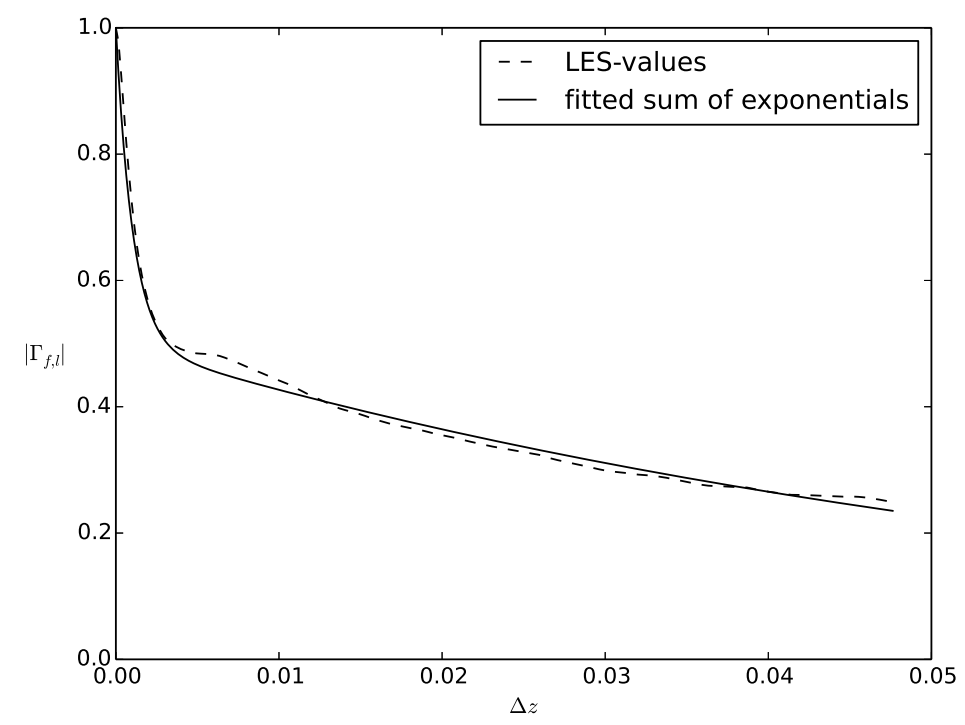

Figure 14: Comparison between absolute value of the force density coherence in the low-frequency range, computed by LES-simulations and the curve-fitted sum of exponentials.

with $m_{\alpha}$ the modal mass, $\omega_{\alpha}$ the modal frequency and $\zeta_{\alpha}$ the modal damping ratio. The displacement spectrum can therefore be written as follows:

$$
\begin{gathered}
S_{d}(z, \omega)=\sum_{\alpha} \sum_{\beta} \phi_{\alpha}^{*}(z) H_{\alpha}^{*}(\omega) J_{\alpha \beta}(\omega) H_{\beta}(\omega) \phi_{\beta}(z), \\
J_{\alpha \beta}(\omega)=\int_{0}^{L} \int_{0}^{L} \phi_{\alpha}^{*}\left(\overrightarrow{z^{\prime}}\right) S_{f}(\Delta z, \omega) \phi_{\beta}\left(\overrightarrow{z^{\prime \prime}}\right) \mathrm{d} z^{\prime} \mathrm{d} z^{\prime \prime},
\end{gathered}
$$

A commonly used approximation to the previous approach is to limit the number of modes. It is not uncommon to use only the ground mode (Curling and Païdoussis, 2003; Chen, 1987), which is also the approach used in this paper. The modal properties can either be computed numerically (De Ridder et al., 2013) or determined from experiments (Chen, 1987). The cylinder is clamped at both sides and the modal parameters used are: $m_{1}=1.19 \mathrm{~kg} / \mathrm{m}, \omega_{1}=175.9 \mathrm{rad} / \mathrm{s}$, $\zeta_{1}=0.012$.

The force density spectrum, discussed in the previous section, cannot be used directly in these formulas because the length of the computational domain is much shorter than the length of the cylinder. Secondly, at frequencies lower than $10 \mathrm{~Hz}$, the scatter might become too large. Therefore, the data are not used directly. Instead, a model is created based on the observations in the previous section.

As Figure $13 \mathrm{~b}$ showed that the force density spectrum obeys the convective nature of fluctuations, the complex part of the proposed model is provided by a complex exponential like in the Corcos model. The second important observation is that at low frequencies, the absolute value becomes independent of frequency.

The absolute value of the force coherence function is then modeled by a sum of decaying exponential terms, which is, unlike in the Corcos model, independent of frequency. The average coherence of the force density is plotted in Figure 14. The average is taken over a frequency range from $12.5 \mathrm{~Hz}$ to $125 \mathrm{~Hz}$, which corresponds to the upper frequency of the constant force spectral density range in Figure 12. The absolute value of the coherence shows a very fast initial decrease and a slower long-range decrease. The reason for this behavior is not entirely clear. Similarly as in the Chase-model, the coherence of the force is fitted with two decaying exponentials, yielding the following model:

$$
\Gamma_{f}=\left(\alpha_{1} e^{-\frac{\Delta z}{\beta_{1} D_{H}}}+\alpha_{2} e^{-\frac{\Delta z}{\beta_{2} D_{H}}}\right) e^{-\frac{\omega \Delta z}{U_{c}} i}
$$




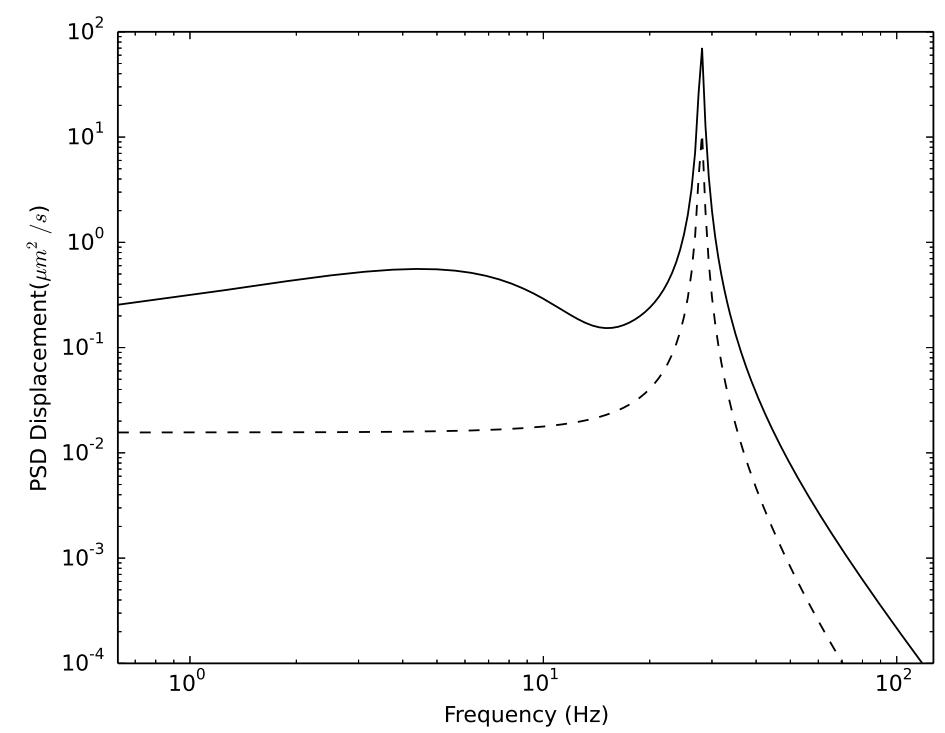

Figure 15: Displacement spectrum halfway along the cylinder computed with a one mode approximation using the empirical spectra by Chen (1987) (solid line) and using the LES-data (dashed line).

The constants are obtained from a curve fit: $\alpha_{1}=\alpha_{2}=0.5, \beta_{1}=5, \beta_{2}=0.08$. The power spectral density used, is the low-frequency limit in Figure 12, namely $1.5 \mathrm{~N}^{2} / \mathrm{m}^{2} \mathrm{~s}$.

The displacement spectrum is computed with the aid of Equations 18,19,20 and is plotted in Figure 15. The peak at $30 \mathrm{~Hz}$ is the resonance peak corresponding to the ground mode frequency of the beam. At higher frequencies, the displacement spectrum decreases fast. The low-frequency range however contributes considerably to the root-mean squares amplitude.

The displacement spectra computed with the aid of the empirical model of Chen (1987) agrees reasonably with the results from the LES-computations, but it predicts a much higher intensity at low frequencies. In later experiments by Wilson and Jones (1983), the pressure power spectral density at low frequencies was seen to level off. This model reduces the gap between the empirical theory and the current calculated values.

The root-mean-square of the amplitude of motion can be computed by

$$
\eta_{r m s}(z)=\sqrt{\int_{0}^{\infty} S_{d}(z, f) \mathrm{d} f} .
$$

According to the empirical model, the rms-value halfway along the cylinder equals $9.6 \mu \mathrm{m}$, which overestimates the measured rms-displacement of $5.8 \mu \mathrm{m}$. The displacement based on the LES-values is $3.7 \mu \mathrm{m}$, which is underestimating the rms-displacement. This can be attributed to the fact that turbulent boundary layer pressure variation is an important contributor to the measured vibration, but some far-field sound-induced vibration may also be present. Secondly, if the measurements of Chen (1987) at higher flow velocities are extrapolated to lower flow velocities, a smaller displacement is expected, rather around $3 \mu m$.

Although the empirical model was shown to be unphysical in the low frequency region, the predicted rms-values have the same order of magnitude as the predictions by LES. In the empirical model the force density is almost completely correlated along the length of the cylinder, with consecutive sign switches, while the computational results showed that the coherence was limited in length. This indicates that the empirical model is not generally valid. 


\section{Conclusions}

Wall-resolved Large Eddy Simulations were used to predict the pressure spectrum induced by turbulent boundary layers on a cylinder in annular flow. The pressure coherence displayed bands, with minima corresponding to a phase angle of $\pi$. The phase angle agreed with the notion that pressure and forces are convected with the mean flow. The pressure coherence did not obey the multiplication hypothesis, which states that the coherence in any given direction can be written as a product of the streamwise and spanwise coherence. The pressure power spectral density agreed well with experimental measurements. At low frequencies it was constant, at $\mathrm{St}=0.5$ it started increasing until it reached a maximum at $\mathrm{St}=3$, after which it decreased.

According to the present computations the force density per unit length showed a maximum in its PSD and constant coherence length at low frequencies. The Corcos model predicts an ever increasing coherence length with decreasing frequency. The Chase model was able to capture a maximum in coherence, however the coherence length decreased too fast at lower frequencies. Above $\mathrm{St}=1$ the computed coherence agreed well with both the Chase and Corcos model.

The force densities were finally used to predict the vibrations induced by a turbulent flow on a cylinder in annular flow. The computed displacement was in reasonable agreement with experimental values. Despite the similarity of the rms-predictions with an empirical model, the computations demonstrated that the latter model is not generally applicable.

\section{Acknowledgments}

The authors gratefully acknowledge the funding by the Research Foundation-Flanders (FWO), through the Ph.D.fellowship of Jeroen De Ridder. The computational resources and services used in this work were provided by the VSC (Flemish Supercomputer Center), funded by Ghent University, the Hercules Foundation and the Flemish Government - department EWI.

\section{Appendix A. Empirical spectra}

\section{Appendix A.1. Empirical power spectral density of pressure}

Chen and Wambsganss (1972) measured the pressure power spectral density in the same annular flow domain as the one used in this paper. They also measured the rms of the resulting vibration. The inner cylinder was a brass cylinder, clamped at both ends, with a length of $1.19 \mathrm{~m}$.

They fitted the following expression to their measurements of the pressure power spectral density:

$$
\begin{array}{ll}
P S D(\omega)=2 \times 0.272 \cdot 10^{-5} S t^{-0.25} & , S t<5, \\
P S D(\omega)=2 \times 22.75 \cdot 10^{-5} S t^{-3} & , S t>5,
\end{array}
$$

where the Strouhal number is $\mathrm{St}=\frac{f D_{h}}{U}$. According to the results of Wilson and Jones (1983) the power spectral density should be constant at St lower than 2.5 .

\section{Appendix A.2. Cross-spectral density}

The empirical expressions considered in this paper are the Corcos and the Chase model. Common to these models is that they assume a statistically homogenous flow. Decaying turbulence from the inlet flow is thus not taken into account. A direct consequence of the homogeneity is that the explicit location vectors $\overrightarrow{x^{\prime}}, \overrightarrow{x^{\prime \prime}}$ in the cross-correlation and the coherence can be replaced by distances; $\zeta_{1}$ the separation in streamwise direction and $\zeta_{2}$ the separation in spanwise direction.

One of the earliest models for the coherence function is the Corcos model, which describes the coherence as an exponentially decaying convective wave:

$$
\Gamma\left(\zeta_{1}, \zeta_{2}, \omega\right)=e^{-\alpha_{1} \frac{\omega \zeta_{1}}{U_{c}}} e^{-\alpha_{2} \frac{\omega \zeta_{2}}{U_{c}}} e^{-\frac{i \omega \zeta_{1}}{U_{c}}} .
$$


In this equation, $U_{c}$ is the convective velocity and $\alpha_{1}, \alpha_{2}$ are empirical coefficients equal to 0.10 and 0.55 respectively. These values are however not unique. The benefit of this model is its simplicity, the drawback is that it fails to predict the low wavenumber region according to Martin and Leehey (1977).

A more fundamental model was developed by Chase (1987). This semi-empirical model is based on a Fourier transform of the pressure Poisson equation. This model contains a part coming from turbulence - mean shear interaction (MT) and one from turbulence - turbulence interaction (TT):

$$
\begin{aligned}
& C S D\left(\zeta_{1}, \zeta_{2}, \omega\right)=S_{p, M T}\left(\zeta_{1}, \zeta_{2}, \omega\right)+S_{p, T T}\left(\zeta_{1}, \zeta_{2}, \omega\right) \\
& S_{p, i}\left(\zeta_{1}, \zeta_{2}, \omega\right)=a_{+} r_{i} \rho^{2} v_{*}^{4} f_{i}\left(\zeta_{1}, \zeta_{2}, \omega\right) e^{\left(-z_{i}-i \omega \zeta_{1} / U_{i}\right)} \\
& f_{M T}\left(\zeta_{1}, \zeta_{2}, \omega\right)=\frac{U_{M T}}{\sqrt{U_{c}^{2}+h_{M T}^{2}}} \alpha_{M T}^{-3} \\
& {\left[1+z_{M T}+\alpha_{M T}^{2} \mu_{M T}^{2}\left(1-z_{M T 1}^{2} / z_{M T}\right)+2 i \alpha_{M T} \mu_{M T} z_{M T 1}\right]} \\
& f_{T T}\left(\zeta_{1}, \zeta_{2}, \omega\right)=\frac{U_{T T}}{\sqrt{U_{c}^{2}+h_{T T}^{2}}} \alpha_{T T}^{-3} \\
& {\left[1+z_{T T}+\alpha_{T T}^{2}\left(1+\mu_{T T}^{2}-\frac{z_{T T 3}^{2}+\mu_{T T}^{2} z_{T T 3}^{2}}{z_{T T}}\right)+2 i \alpha_{T T} \mu_{T T} z_{T T 1}\right]}
\end{aligned}
$$

The equations above represent the corrected version of the Chase model by Josserand and Lauchle (1989). The parameters in this model are given by

$$
\begin{aligned}
& r_{T T}=\frac{C_{T T} h_{T T}}{C_{T T} h_{T T}+C_{M T} h_{M T}}, r_{M T}=1-r_{T T}, \\
& a_{+}=\frac{2 \pi}{3}\left(C_{T T} h_{T T}+C_{M T} h_{M T}\right), \mu_{i}=\frac{h_{i} v_{*}}{\sqrt{U_{c}^{2}+h_{i}^{2} \nu_{*}^{2}}}, \\
& U_{i}=\frac{U_{c}}{1-\mu_{i}^{2}}, \alpha_{i}^{2}=\frac{1}{1-\mu_{i}^{2}}+\frac{U_{i}^{2}}{\omega^{2} b_{i}^{2} \delta^{2}}, \\
& z_{i 1}=\mu_{i} \alpha_{i} \omega \zeta_{1} / U_{i}, z_{i 2}=\alpha_{i} \omega \zeta_{2} / U_{i}, \\
& z_{i}=\sqrt{z_{i 1}^{2}+z_{i 2}^{2}}, h_{i}=\mu_{i} U_{c} / v_{*}
\end{aligned}
$$

with $v_{*}$ the friction velocity and $\delta$ the height of the boundary layer. Although the coefficients $C_{i}, h_{i}$ and $b_{i}$ are not universal, some typical values are provided below

$$
\begin{aligned}
& h_{i}=3, C_{M T}=0.1553, C_{T T}=0.004667, \\
& b_{M T}=0.756, b_{T T}=0.378
\end{aligned}
$$

This model is a big improvement compared to the Corcos model and it actually fulfills the inviscid Kraichnan-Philips theorem, which states that the cross-spectral density should go quadratically to zero as the wavenumber is going to zero. However, in experiments it is observed that at low wavenumbers the cross-spectral density is not going to zero, but that a uniform spectrum exists at low wavenumber (wavenumber-white spectrum (Chase, 1980)).

Bailey, S. C. C., Hultmark, M., Smits, A. J., Schultz, M. P., 2008. Azimuthal structure of turbulence in high Reynolds number pipe flow. Journal of Fluid Mechanics 615, 121-138.

Benhamadouche, S., Moussou, P., Le Maitre, C., 2009. CFD estimation of the flow-induced vibrations of a fuel rod downstream a mixing grid. Proceedings of the 2009 ASME Pressure Vessels and Piping Conference 2009. Fluid-Structure Interaction, 189-197.

Boersma, B. J., Breugem, W. P., 2011. Numerical simulation of turbulent flow in concentric annuli. Flow Turbulence and Combustion 86 (1), $113-127$.

Bonness, W. K., Capone, D. E., Hambric, S. A., 2010. Low-wavenumber turbulent boundary layer wall-pressure measurements from vibration data on a cylinder in pipe flow. Journal of Sound and Vibration 329 (20), 4166-4180. 
Bose, S. T., Moin, P., You, D., 2010. Grid-independent large-eddy simulation using explicit filtering. Physics of Fluids 22 (10), 105103.

Bull, M. K., 1967. Wall-pressure fluctuations associated with subsonic turbulent boundary layer flow. Journal of Fluid Mechanics 28, 719-754.

Bull, M. K., 1996. Wall-pressure fluctuations beneath turbulent boundary layers: Some reflections on forty years of research. Journal of Sound and Vibration 190 (3), 299-315.

Chase, D. M., 1980. Modeling the wavevector-frequency spectrum of turbulent boundary layer wall pressure. Journal of Sound and Vibration 70 (1), 29-67.

Chase, D. M., 1987. The character of the turbulent wall pressure spectrum at subconvective wavenumbers and a suggested comprehensive model. Journal of Sound and Vibration 112 (1), 125-147.

Chase, D. M., 1993. A semiempirical model for the wave-vector-frequency spectrum of turbulent wall-shear stress. Journal of Fluids and Structures 7 (6), 639-659.

Chen, S. S., 1987. Flow-induced vibration of circular cylindrical structures. Hemisphere Pub. Corp.

Chen, S. S., Wambsganss, M. W., 1972. Parallel-flow-induced vibration of fuel rods. Nuclear Engineering and Design 18 (2), $253-278$.

Choi, H., Moin, P., 2012. Grid-point requirements for large eddy simulation: Chapman's estimates revisited. Physics of Fluids 24 (1).

Chung, S. Y., Rhee, G. H., Sung, H. J., 2002. Direct numerical simulation of turbulent concentric annular pipe flow - Part 1: Flow field. International Journal of Heat and Fluid Flow 23 (4), 426-440.

Ciappi, E., Magionesi, F., De Rosa, S., Franco, F., 2012. Analysis of the scaling laws for the turbulence driven panel responses. Journal of Fluids and Structures 32, 90-103.

Corcos, G. M., 1964. The structure of the turbulent pressure field in boundary-layer flows. Journal of Fluid Mechanics 18 (3), $353-378$.

Curling, L. R., Païdoussis, M. P., 1992. Measurements and characterization of wall-pressure fluctuations on cylinders in a bundle in turbulent axial flow; part 1: Spectral characteristics. Journal of Sound and Vibration 157 (3), 405-433.

Curling, L. R., Païdoussis, M. P., 2003. Analyses for random flow-induced vibration of cylindrical structures subjected to turbulent axial flow. Journal of Sound and Vibration 264 (4), 795-833.

De Ridder, J., Degroote, J., Van Tichelen, K., Schuurmans, P., Vierendeels, J., 2013. Modal characteristics of a flexible cylinder in turbulent axial flow from numerical simulations. Journal of Fluids and Structures 43, 110-123.

Evans, N. D., Capone, D. E., Bonness, W. K., 2013. Low-wavenumber turbulent boundary layer wall-pressure measurements from vibration data over smooth and rough surfaces in pipe flow. Journal of Sound and Vibration 332 (14), 3463-3473.

Gagnon, J. O., Païdoussis, M. P., 1994. Fluid coupling characteristics and vibration of cylinder cluster in axial flow. part i: Theory. Journal of Fluids and Structures 8 (3), 257-291.

Gerolymos, G. A., Senechal, D., Vallet, I., 2013. Wall effects on pressure fluctuations in turbulent channel flow. Journal of Fluid Mechanics 720, $15-65$.

Gloerfelt, X., Berland, J., 2013. Turbulent boundary-layer noise: direct radiation at mach number 0.5. Journal of Fluid Mechanics 723, 318-351.

Graham, W. R., 1997. A comparison of models for the wavenumber-frequency spectrum of turbulent boundary layer pressures. Journal of Sound and Vibration 206 (4), 541-565.

Hambric, S. A., Hwang, Y. F., Bonness, W. K., 2004. Vibrations of plates with clamped and free edges excited by low-speed turbulent boundary layer flow. Journal of Fluids and Structures 19 (1), 93-110.

Josserand, M. A., Lauchle, G. C., 1989. Cross-spectral density of the wall pressure fluctuations under a turbulent boundary layer. Journal of Sound and Vibration 128 (3), 519-522.

Lee, Y. T., Blake, W. K., Farabee, T. M., 2005. Modeling of wall pressure fluctuations based on time mean flow field. Journal of Fluids EngineeringTransactions of the ASME 127 (2), 233-240.

Martin, N. C., Leehey, P., 1977. Low wavenumber wall pressure measurements using a rectangular membrane as a spatial filter. Journal of Sound and Vibration 52 (1), 95-120.

Meneveau, C., Lund, T. S., Cabot, W. H., 1996. A lagrangian dynamic subgrid-scale model of turbulence. Journal of Fluid Mechanics 319 , 353-385.

Moreno, M. M., 2000. Simulation numérique des efforts aléatoires exercés par un écoulement turbulent annulaire. Ph.D. thesis, Université Paris VI.

Nouri, J. M., Umur, H., Whitelaw, J. H., 1993. Flow of Newtonian and non-Newtonian fluids in concentric and eccentric annuli. Journal of Fluid Mechanics 253, 617-641.

Ohlmer, E., Russo, S., Schwemmle, R., 1972. Investigation of an analytical model for parallel flow induced rod vibrations. Nuclear Engineering and Design 22 (2), 272-289.

Païdoussis, M. P., 2004. Fluid-Structure Interactions: Slender Structures and Axial Flow. Academic Press.

Peltier, L. J., Hambric, S. A., 2007. Estimating turbulent-boundary-layer wall-pressure spectra from CFD RANS solutions. Journal of Fluids and Structures 23 (6), 920-937.

Sillero, J. A., Jimenez, J., Moser, R. D., 2014. Two-point statistics for turbulent boundary layers and channels at reynolds numbers up to delta(+) approximate to 2000. Physics of Fluids 26 (10).

Smol'yakov, A. V., 2006. A new model for the cross spectrum and wavenumber-frequency spectrum of turbulent pressure fluctuations in a boundary layer. Acoustical Physics 52 (3), 331-337.

Willmarth, W. W., Winkel, R. E., Sharma, L. K., Bogar, T. J., 1976. Axially-symmetric turbulent boundary-layers on cylinders - mean velocity profiles and wall pressure-fluctuations. Journal of Fluid Mechanics 76, 35-64.

Willmarth, W. W., Yang, C. S., 1970. Wall-pressure fluctuations beneath turbulent boundary layers on a flat plate and a cylinder. Journal of Fluid Mechanics 41, 47-80.

Wilson, R. J., Jones, B. G., 1983. Turbulent pressure-velocity measurements in a fully-developed concentric annular air-flow. Journal of Vibration Acoustics Stress and Reliability in Design-Transactions of the ASME 105 (3), 345-354. 